\title{
Tweetchats, Disseminating Information, and Sparking Further Scientific Discussion with Social Media
}

\author{
Michael A. Chary ${ }^{1}$ (1) $\cdot$ Peter R. Chai ${ }^{2,3,4,5}$ \\ Received: 3 December 2019 / Revised: 2 February 2020 / Accepted: 2 February 2020 /Published online: 24 February 2020 \\ (C) American College of Medical Toxicology 2020
}

Tweetchats and tweetorials can provide a wider forum for scholarly discussion than in-person journal clubs or printed commentaries. However, social media can distort conversations, selectively amplifying comments that curry favor rather than substantive ones. In 280 characters, it is easier to virtue signal than communicate a complex or subtle thought. Curating tweetchats and tweetorials (collectively tweet threads) as well as linking social media discourse to primary sources provides an antidote to this distortion and leaves us with Twitter as a record of discourse that is usually not written down. Tweet threads do not replace thoughtful detailed analysis. They do record the integration of new research findings into received medical knowledge.

Tweetchats and tweetorials are two ways of grouping tweets on Twitter. A tweetchat is a scheduled multi-person Twitter chat centered on a specific topic. A tweetorial is a collection of thematically related tweets, a twitter version of a tutorial. The distinction between tweetchats and tweetorials is blurry and I refer to both of them as tweet threads. Tweets for a tweetorial may be grouped together by using a hashtag, just like tweets for a tweetchat, or each tweet in a tweetorial may be a reply to an earlier tweet. Tweet threads can draw

Supervising Editor: Mark B. Mycyk, MD

Michael A. Chary

Michael.Chary@childrens.harvard.edu

1 Regional Center for Poison Control and Prevention Serving Massachusetts and Rhode Island, Boston, USA

2 Division of Medical Toxicology, Department of Emergency Medicine, Harvard Medical School, Brigham and Women's Hospital, Boston, MA, USA

3 The Fenway Institute, Boston, MA, USA

4 The Koch Institute for Integrated Cancer Research, Massachusetts Institute of Technology, Cambridge, MA, USA

5 Massachusetts and Rhode Island Regional Center for Poison Control and Prevention, Boston, MA, USA attention to scholarly work that might otherwise be missed or lost in the deluge of daily publications. Anyone with access to Twitter can view the discussion in real time and (re)visit the thread later. Residencies in psychiatry [1], surgery [2, 3], infectious disease [4], and emergency medicine [5] have used tweetchats and tweetorials to create asynchronous journal clubs.

Over the last two years, the American College of Medical Toxicology (ACMT) and editorial board of the Journal of Medical Toxicology (JMT) conducted quarterly tweetchats (\#firesidetox) to discuss manuscripts published in JMT. For two recent \#firesidetox tweetchats, JMT collaborated with its publisher Springer Nature to make the featured articles freely available via SharedIt for the week before and week after the tweetchat. These two tweetchats involved 23 and 26 accounts (more than one person can use the same account simultaneously), from the United States, Australia, Poland, and Qatar, and spanned 150 tweets [6].

Tweet threads harken back to when scientific communications were letters among colleagues. History does indeed rhyme. Tweets, however, are shorter than letters. Each tweet can only be 280 characters, favoring succinct perhaps simplistic statements that are easily read on a mobile device [7]. These quips may capture important criticism, support, or background information for manuscripts. Reading these tweets does not replace reading the full manuscript.

Alternative metrics (Altmetrics), such as the number of tweets, are designed to assess the impact of scholarly work via social media, which the canonical metric of article citations overlooks [8]. Altmetrics include counts of web page views, PDF downloads, comments posted on the journal web site, blogs, or web sites such as Wikipedia, Twitter, Facebook, ResearchGate, and Academia.edu as well as the number of saves to online citation managers such as Mendeley, Zotero, and CiteULike. Alternative metrics such as saves, like retweets, favor quantity and immediate activity over quality and may not necessarily reflect sustained engagement.. 
Twitter-based altmetrics (number of retweets, likes, or comments a post receives) can bias which tweets drive a conversation. Three quick tweets, each one a smiley face, count three times as much as one thoughtful tweet. Participants remain invisible unless they retweet, like, or post a comment. Each post becomes a race for likes, retweets, or virtue signaling at the expense of critical thought or reflection [9]. This can encourage gamesmanship and sensationalism at the expense of reasoned debate [10]. The spread of fake news and of the concern about the impact of fake news demonstrates the difficulty in verifying complex statements in real time and the lingering effect that even debunked tweets can have.

How much altmetrics and journal citations measure the same type of impact is an open issue. Journals that have their own Twitter account obtain more tweets but not more citations than journals without a dedicated account [11]. For the Journal of Medical Internet Research, the number of tweets predicted the number of citations in the first three days after publication [12], even though many of these tweets were duplicates or created by automated accounts (bots). The number of tweets an ecology article receives predicts the number of citations it will receive more accurately than the 5-year impact factor of the journal in which it was published [13]. There is no reported relationship between highly cited and highly tweeted articles in molecular biology [14] or public health [15].

Alternative metrics can provide a fuller view of a researcher's impact by including information distinct from citations. GitHub and Bitbucket are online repositories where computational researchers post source code. For each project, GitHub tracks how many people have used the source code in another project, how many people have commented on the code, and how many people are watching the evolution of the repository. These metrics motivate computational researchers to be more transparent. I am more likely to cite the paper of someone whose code I can see, understand, and extend.

Activity on Twitter is, nevertheless, more used and more generally applicable than niche measures that track software use. The challenge for the academic physician is to use Twitter to articulate a pointer to a more articulate thought. Commenting with images or short movies gets around the information ceiling of 280 characters, even if images shape our perception in other ways. Images can powerfully convey an impression and avoid the temptation to argue fine points with out-of-context quotes. Linking to primary material and other web discussions may prevent the tweetchat or tweetorial from becoming an echo chamber. Web sites, such as Stack Exchange, Reddit, or ResearchGate host discussions on scientific topics, but usually not specific papers. arXiv and bioRxiv are publicly accessibly servers to which researchers can upload preliminary drafts of manuscripts (not peerreviewed, not copy-edited) to share. Tox and the Hound, EM:RAP, and many \#FOAM (free open-access medical education) blogs provide longer form audio and written tutorials.
Academic physicians should recognize the limited role that activity on social media currently plays in promotion along the tenure track - moving from instructor to assistant professor to associate professor to full professor - and balance their engagement with social media accordingly. The most accepted metrics for scholarly impact are the number of publications or grants, not retweets or followers. Universities might consider activity on social media more in their decisions to promote a physician along the tenure track if research demonstrates that activity on social media improves unequivocal measures of scholarship. Some academic institutions have guidelines for assessing the scholarly value of activity on social media for promotion [16].

Curating tweet threads could have scholarly value [17], producing a guided digest similar to Cosma Shalizi's online notebooks. Curating a tweet refers to responding to that tweet, after the tweetorial or tweetchat has finished, with a tweet that clarifies, provides, or links to more detail. Curating tweets gives a remedy against the necessarily incomplete nature of the immediate response and provides a means to periodically refresh the link between Twitter and in real-life discussions.

Twitter may also have scholarly value as a conduit for knowledge transfer. Consider organizing threads around \#ACMT2020, \#ACEP2020, or \#choosingwisely. Polls could be embedded within tweetorials or tweetchats to assess comprehension or how likely the reader is to change his or her practice. These polls could operate longitudinally with minimal effort, potentially reaching a larger audience than traditional approaches. The Twitter account engagement with ACMT's recent tweetchats $(n=23,26)$, even though they were only open for 90 minutes, suggests that barriers to realizing a larger sample size still exist.

Participants in tweetchats must be mindful of institutional policies on social media. Tweets are publicly available by default. There is a risk of inadvertently disclosing protected health information while discussing rare medical toxicology exposures. Fear of this risk should not halt discussion. One should follow journal and institutional standards for de-identification. Perhaps the patient(s) or those who make health care decisions for the patient could be invited to join, turning concern over privacy and the public-facing nature of these discussions into strength rather than a liability. Prior tweetchats on suicide prevention [18] and breast cancer patient education [19] included the public, clinicians, and scientists, although neither tweetchats discussed a specific case. Discussion should be halted if protected health information is inadvertently disseminated despite following institutional policies.

Tweeting by medical toxicologists can extend the traditional means of discussing and disseminating scholarly work. Tweet threads can embed graphics and polls to allow deeper discussion and ascertain impact. Curating tweet threads can stem Twitter's tendency to amplify clickbait. Academic physicians should recognize that some but not all appointments and promotion committees' universities consider social media activity alongside accepted measures of scholarship (grants, publications). 
Communication on social media should be done mindful of but not fearful of institutional policies. Activity on social media should be a means to the end of creating impressions and linking discussions for academic physicians.

Funding Information PRC is funded by NIH K23DA044874, R01DA047236, Gilead Sciences, and the Hans and Mavis Lopater Psychosocial Foundation. MAC is funded by the NIDA/NIH Loan Repayment Program.

\section{Compliance with Ethical Standards}

Conflict of Interest None.

\section{References}

1. Peters ME, Uible E, Chisolm MS. A Twitter education: why psychiatrists should tweet. Curr Psychiatry Rep. Springer. 2015;17:94.

2. Logghe HJ, Selby LV, Boeck MA, Stamp NL, Chuen J, Jones C. The academic tweet: Twitter as a tool to advance academic surgery. J Surg Res. Elsevier. 2018;226:viii-xii.

3. Luc JGY, Varghese TK Jr, Antonoff MB. Participating in a TweetChat: practical tips from the thoracic surgery social media network (\# TSSMN). Ann Thorac Surg Elsevier. 2019;107:e229 33.

4. Goff DA, Kullar R, Newland JG. Review of twitter for infectious diseases clinicians: useful or a waste of time? Clin Infect Dis Oxford University Press. 2015;60:1533-40.

5. Thoma B, Mohindra R, Artz JD, Chan TM. CJEM and the changing landscape of medical education and knowledge translation. CJEM. Cambridge University Press. 2015;17:184-7.

6. Chai PR, Ruha A-M, Wong KE, Monette DL, Spyres MB, Lapoint $\mathrm{J}$, et al. The virtual toxicology journal club: the dissemination and discussion of noteworthy manuscripts using twitter. J Med Toxicol. 2018;14:212-7.

7. Ott BL. The age of twitter: Donald J. Trump and the politics of debasement. Crit Stud Media Commun. Routledge. 2017;34:59 68. https://doi.org/10.1080/15295036.2016.1266686.

8. Cabrera D, Roy D, Chisolm MS. Social media scholarship and alternative metrics for academic promotion and tenure. J Am Coll
Radiol. 2018;15:135-41 Available from: http://www.sciencedirect. com/science/article/pii/S1546144017311341.

9. Binns A, Bateman M. And they thought Papers were Rude. Br J Rev. SAGE Publications Sage UK: London, England. 2018;29:3944.

10. Merchant RM, Asch DA. Protecting the value of medical science in the age of social media and "fake news". JAMA. 2018;320:2415-6.

11. Ortega JL. The presence of academic journals on twitter and its relationship with dissemination (tweets) and research impact (citations). Aslib J Inf Manag Emerald Publishing Limited. 2017;69: 674-87.

12. Eysenbach G. Can tweets predict citations? Metrics of social impact based on twitter and correlation with traditional metrics of scientific impact. J Med Internet Res. 2011;13:e123 Available from: http:// www.ncbi.nlm.nih.gov/pubmed/22173204.

13. Peoples BK, Midway SR, Sackett D, Lynch A, Cooney PB. Twitter predicts citation rates of ecological research. PLoS One. Public Library of Science. 2016;11:e0166570.

14. Zhang L, Wang J. Why highly cited articles are not highly tweeted? A biology case. Scientometrics. 2018;117:1-15.

15. Tonia T, Van Oyen H, Berger A, Schindler C, Künzli N. If I tweet will you cite? The effect of social media exposure of articles on downloads and citations. Int J Public Health. 2016;61:513-20 Available from: https://ideas.repec.org/a/spr/ijphth/ v61y2016i4d10.1007 s00038-016-0831-y.html.

16. Cabrera D, Vartabedian BS, Spinner RJ, Jordan BL, Aase LA, Timimi FK. More than likes and tweets: creating social media portfolios for academic promotion and tenure. J Grad Med Educ. 2017;9:421-5. https://doi.org/10.4300/JGME-D-17-00171.1.

17. Wang AT, Sandhu NP, Wittich CM, Mandrekar JN, Beckman TJ. Using social media to improve continuing medical education: a survey of course participants. Mayo Clin Proc. 2012;87:1162-70.

18. McVey N, Fillingham J, Owen H. Suicide prevention: a planned approach to discussing a sensitive topic through tweetchats. Physiotherapy Elsevier. 2016;102:e131-2.

19. Attai DJ, Cowher MS, Al-Hamadani M, Schoger JM, Staley AC, Landercasper J. Twitter social media is an effective tool for breast cancer patient education and support: patient-reported outcomes by survey. J Med Internet Res. JMIR Publications Inc., Toronto, Canada. 2015;17:e188.

Publisher's Note Springer Nature remains neutral with regard to jurisdictional claims in published maps and institutional affiliations. 